\title{
Structural Analysis of High Frequency Parameters for Discrete Components
}

\author{
T.Pearson, M.Madheswaran, Member, IEEE
}

\begin{abstract}
There can be various EMC expert systems developed for analyzing the PCB. The algorithms used to predict possible radiated emissions problems from a printed circuit board have been presented. From accuracy point of view, the expert system approach is approximately equal to human EMC expert with a through knowledge of the board and a calculating aid. The algorithms also make assumptions and approximations about how the board will interact with the rest of the system.
\end{abstract}

\section{Index Terms-EMC, EMS, Standards, Expert System.}

\section{INTRODUCTION}

Electromagnetic compatibility (EMC) often refers to one of the mysteries in the field of electrical and electronic engineering, as the EMC behavior of a product is unpredictable. In most cases, the EMC performance of the product cannot be found precisely with the highest level of simulation tools [1]. No single simulation software covers all the parameters and the rules governing the EMC design. Most of the engineers rely on measurement and testing methods after the prototype of the product is fabricated. In large-scale electrical and electronic projects, the end result may be catastrophic when there are critical EMC problems identified after the completion of infrastructure

\section{HISTORY}

In the early days of electrical engineering in 1800s, all electrical systems were used for heating, lighting and machinery driving were controlled by simple on/off switches. EMC problems did not appear in these simple systems. A break through on the semiconductor technology in 1970s significantly increased the EMI problem. High frequency switching elements began to be widely applied in power, digital control and communication systems. Source of EMI was not only limited to simple switching but also to a wideband, high frequency and high power source. Furthermore, the digital control circuits were widely adopted, which were more vulnerable towards the noise. A switching power supply was a typical example of the EMI problem at

Manuscript received May 31, 2009.

T.Pearson, is with the Department of Electronics and Communication Engineering, SMK Fomra Institute of Technology, Chennai,Tamilnadu,603103, India, Phone : +91 44 27475621; Fax :+ 9144 2747522; e-mail : pearsonin@gmail.com

M.Madheswaran, is with the Department of Electronics and Communication Engineering, Muthayammal Engineering College Rasipuram , Namakkal District, Tamilnadu, 637 408, India, e-mail : madheswaran.dr@gmail.com this stage when it comprises both high power switching and digital control circuits. Engineers could no longer rely on the traditional techniques to solve EMI problems. New tactics were developed using shielding and filtering and using border team electromagnetic compatibility (EMC) between products was defined.

The EMI reduction techniques have evolved over three decades. They are used as general design rules instead of precise calculations. At the beginning of the 21st century, new EMC reduction techniques have been developed to direct towards the integration approach with full computer simulation technologies. Making use of computer simulation together with artificial intelligence optimized EMC solutions can be generated automatically. Before a full set of automatic EMC solution is available, research should focus on the solution of individual EMC parameters using the de-coupled method. Hopefully these solutions should be integrated into an ultimate solution in the future.

Although there are many computer modeling tools are available, those are rarely being used for analyzing the PCB's. Computer modeling can provide valuable insight to a board designer as critical circuits are being placed and routed, but they are not good at identifying the unintentional emissions sources and coupling paths that result most EMC problems. Full wave modeling of printed circuit board is not a practical option considering the complexity of the latest technology. Even with infinite computational resources, the board designer would not have all the necessary information about the components, signals and software to do an accurate analysis. Further the EMI test procedures have repeatability issues that prevent their results from being accurately predicted by computer algorithms.

\section{THE PROBLEM}

Following rapid development of science and technology, human society has entered into a new era of information technology and communication in the 21 st century. Today, the whole environment is surrounded by the significant levels of background electromagnetic noise emitted from mobile communication networks, computers, microprocessors, electrical appliances and so on. It is estimated that there is a $7 \%$ - $14 \%$ increase in the background.

The severe electromagnetic noise environment is hazardous towards electrical and electronic equipment, especially valuable electronic control system. The electromagnetic hazard is a cause for great concern around the world. A world-wide study and research are then initiated for electromagnetic noise reduction techniques. Recent research not only concentrates on electromagnetic noise 
reduction, but also on compatibility between equipment and environment in electromagnetic aspects. Therefore a broader term electromagnetic compatibility (EMC) is referred to, to cover the wider scope. This means that the electrical/electronic equipment is compatible with each others without any deterioration in their function within a defined time and space.

EMC refers to two aspects, electromagnetic interference (EMI) and electromagnetic susceptibility (EMS). EMI refers to the noise level generated by the equipment, which causes interference with others while EMS refers to the immunity of equipment against electromagnetic noise from the environment. Equipment with good electromagnetic compatibility emits minimum noise to the environment as well as being able to resist the noise from the environment.

The existence of electromagnetic compatibility requires three conditions:

a) Equipment to generate electromagnetic noise - (source)

b) Equipment to receive electromagnetic interference - (victim)

c) Media for the electromagnetic noise - (comply with)

Their relationship is illustrated in Figure 1.

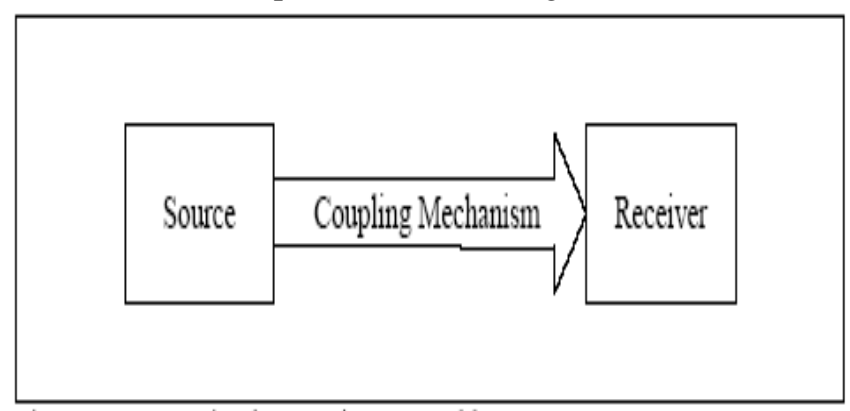

Fig. 1. Basic Elements in EMI Problems

The coupling path is the emission path of the electromagnetic noise. It is equivalent to the transmission of electromagnetic energy. Moreover, it is divided into conducted emission and radiated emission. Conducted emission requires certain transmission media but radiated emission does not require any. Radiated emission is classified into the near field and far field depending on the transmission distance $\mathrm{d}$ and the wavelength of the transmission wave $\lambda$. Generally, the transmission wave with $\mathrm{d}<\lambda / 2 \pi$ refers to near field while $\mathrm{d}<\lambda / 2 \pi$ refers to far field.

Near field interference can be further divided into electric field and magnetic field interference, which are commonly used as capacity interference and inductive interference respectively. Far field refers to the transmission of electromagnetic energy in free space, which is commonly used as radiation interference. The illustration in the Figure 2 shows a hierarchy of the coupling path of electromagnetic interference.

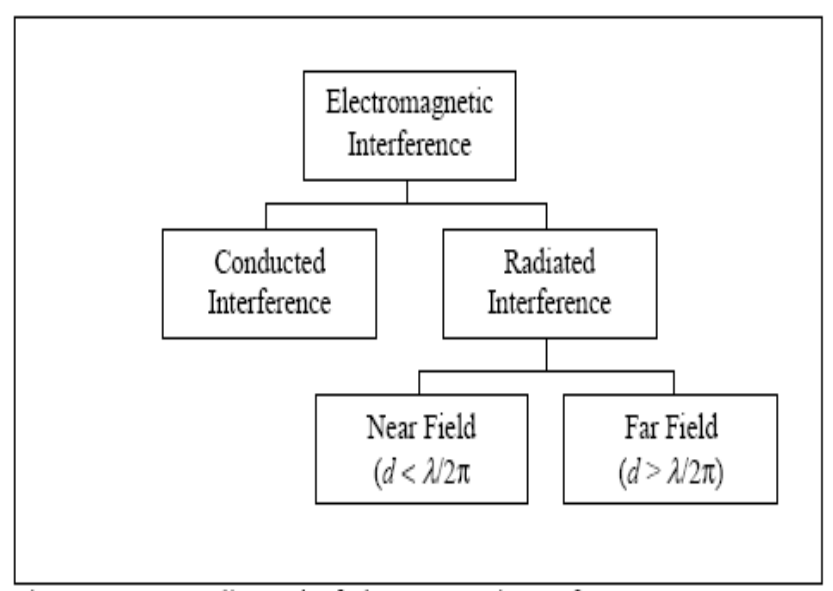

Fig. 2. Coupling Path of Electromagnetic Interference

Electromagnetic interference relies on the following necessary elements, namely the source, comply path and victim. All suppression methods focus on either one of these factors such as suppressing the electromagnetic noise from the source, removing/reducing the coupling path and improving electromagnetic immunity of the victim.

\section{EMC STANDARDS}

In order to ensure a good electromagnetic environment and the compatibility between different electrical/electronic equipment, many countries and related international associations had defined a series of international standard for EMC, such as the US Federal Communications Commission (FCC), the International Electro-technical Commission (IEC and CISPR), the European Committee for Electro-technical Standardization (EN), the US Military Standard in EMC (MIL-STD-460), the Germany National Standard (VDE), and the French Standard (NFF) etc.

Any scientific measure on EMC of particular electrical/electronic equipment should rely on testing. This can verify the performance of the equipment in terms of EMC against international EMC standards. In accordance with the classifications of EMC, the tests are divided into emissions tests and susceptibility test. The emission test can be further divided into conducted emission test and a radiation emission test. Moreover, tests are developed in each stage of product design cycle i.e. research stage, breadboard stage, pre-fabrication stage, type-test stage, etc. The requirement for EMC testing equipment, testing method and the surrounding environment shall be deliberately defined before any tests are carried out. Figure 3 classifies the EMC testing.

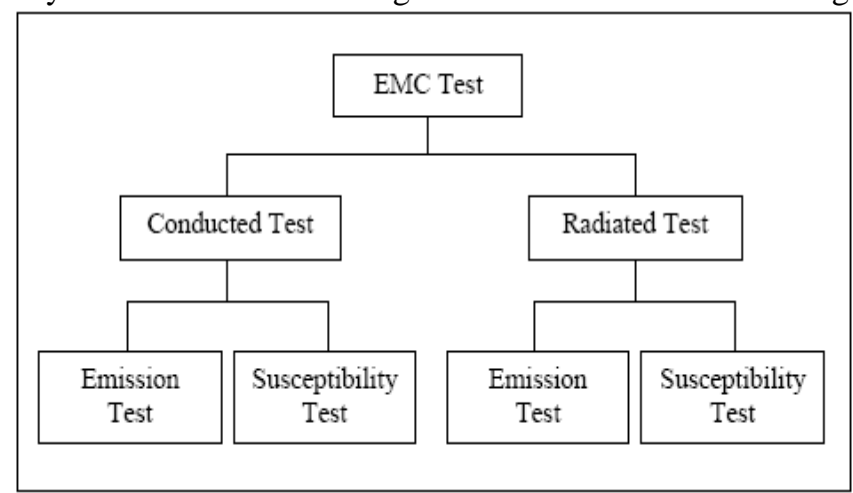

Fig. 3. EMC testing category 


\section{EXPERT SYSTEM}

There can be various EMC expert systems developed for analyzing the PCB. One of the EMC expert system developed for PCB generally consists of four stages as listed below [2].
1. Input
2. Classification
3. Evaluation
4. Reporting

The board layout and component input data, the characteristics of all the net list and their signals are identified in the net classification stage. This information is passed to evaluate algorithms, which search for possible radiation or susceptibility problems. The basic structure of the PCB EMC expert system is shown in figure 4.

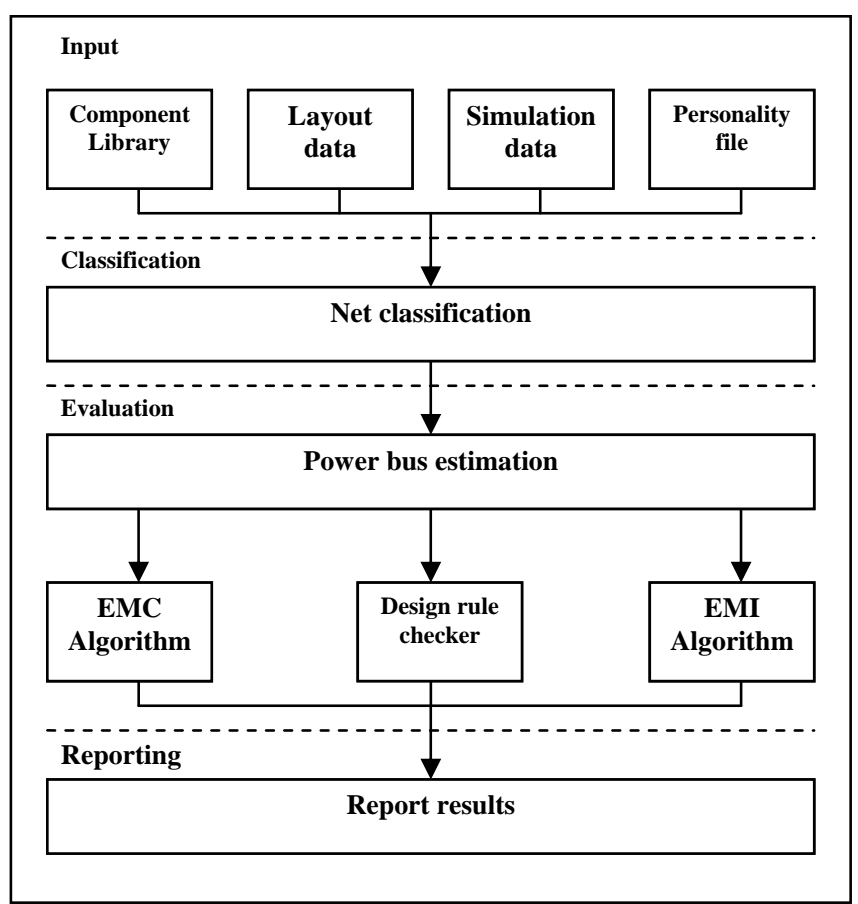

Fig. 4. Structure of the PCB EMC expert system

\section{Differential Mode Radiation Algorithm}

This algorithm models signal trace segments and their corresponding return trace segments as current loop radiation sources as illustrated in the figure 5 .

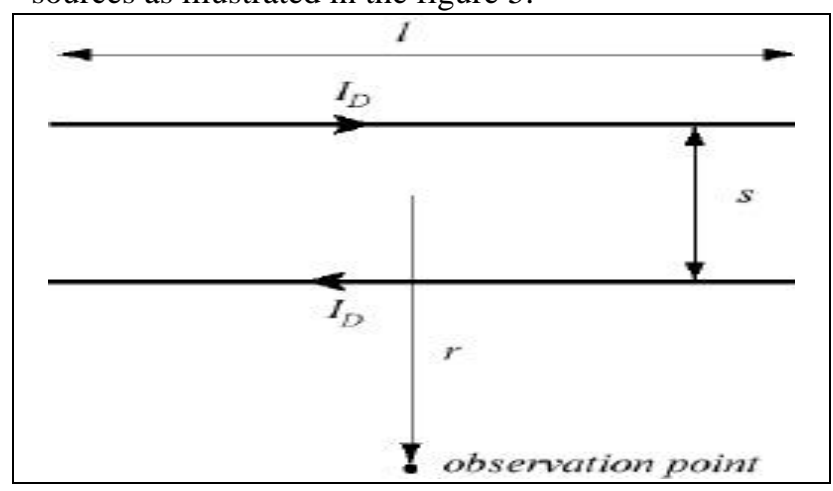

Fig. 5. A simple differential mode current radiation source

The maximum electric filed is given by the formula,

$|\mathrm{E}| \max =1.316 \times 10^{-14}\left|\mathrm{I}_{\mathrm{D}}\right| \mathrm{f}^{2} 1 \mathrm{~s} / \mathrm{r}$ where

$\mathrm{f}$ - frequency in $\mathrm{Hz}$

1 - length of a segment

$\mathrm{s}$ - distance between trace and return trace

$I_{D}$ - magnitude of the return current

$\mathrm{r}$ - radius of the electric field intensity (generally considered as $3 \mathrm{~m}$ )

Hence simplifying the equation we get,

$$
|\mathrm{E}|_{\max }=4.4 \times 10^{-15}\left|\mathrm{I}_{\mathrm{D}}\right| \mathrm{f}^{2} \mathrm{Ld}
$$

Since most EMI regulations require measurements in a semi-anechoic environment, the electric field is multiplied by a factor of two to account for the worse case of reflection off the floor. Therefore the final equation can be written as,

$$
\begin{aligned}
& |\mathrm{E}|_{\max }=4.4 \times 10^{-15}\left|\mathrm{I}_{\mathrm{D}}\right| \mathrm{f}^{2} \mathrm{Ld} \times 2 \\
& |\mathrm{E}|_{\max } \approx 10^{-14}\left|\mathrm{I}_{\mathrm{D}}\right| \mathrm{f}^{2} \mathrm{Ld}
\end{aligned}
$$

Each segment of every net on the circuit board is evaluated by this algorithm[6] at each frequency of interest. The following assumptions are made for the computational analysis. All dimensions are small compared to the wavelength of interest. Segments are thin relative to their spacing and length. Currents on both wires are equal and opposite. When the return path is actually in a plane, the image of the signal trace is used. This assumes that the plane is large relative to the length of the trace segment and the height of the segment above the plane. No phase information is included. Estimate may be high, since actual radiation from many wire segments may not add in phase. Polarity of radiated field is not considered. Estimate may be high, since actual radiation from many wire segments may not have the same polarity are used.

The length, 1, of segment is obtained from layout data file. The distance, s, between trace and return path and the magnitude of differential current, ID, are determined by the subroutines in the net classification stage. Each segment of every net on the board is passed to this algorithm. The routine first checks if the net is a digital net with appropriate utilization or an analog net with a narrow-band signal. If this condition is satisfied, the routine calculates the E-field equation at each frequency. Contributions from each segment are summed linearly to obtain the total E-field radiated by a net.

The differential emission estimate for the entire board is obtained by taking a root mean square sum of the fields for each net as,

$|E|_{\text {total }}=\sqrt{ }\left(E_{\text {seg } 1}\right)^{2}+\left(E_{\text {seg } 2}\right)^{2}+\ldots+\left(E_{\text {seg s }}\right)^{2}$

\section{EXPERT SYSTEM ANALYSIS OF THE TEST BOARD}

A multilayer test board is used to evaluate the expert system algorithms experimentally. This test board shown in figure 6 , is developed for a study of the effects of layer spacing and dielectric materials on radiation algorithms. 


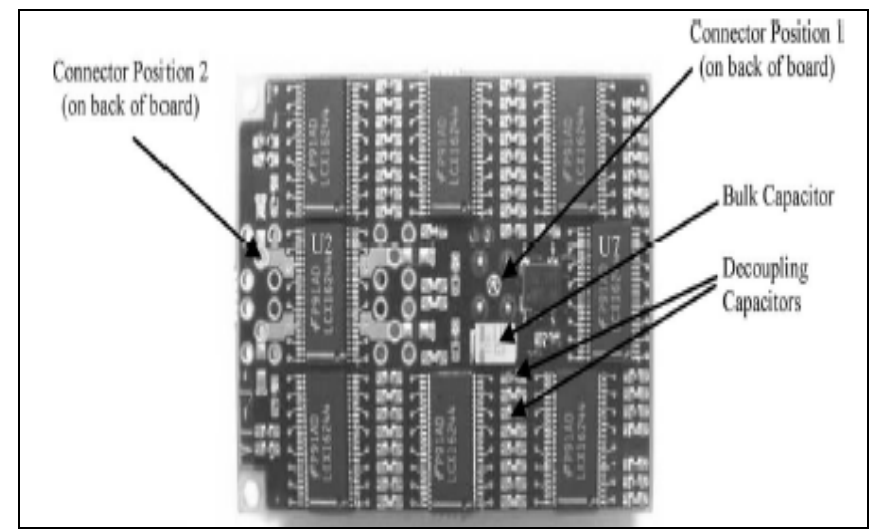

Fig.6. Layout of the test board.

The following diagram in the figure 7 illustrates layer stack up of the test board.

\begin{tabular}{|cl|}
\hline$\frac{0.5-\mathrm{mm} \text { dielectric }}{0.13-\mathrm{mm} \text { dielectric }}$ & L1: Component and surface pads \\
\hline $0.05-\mathrm{mm}$ dielectric & L2: Signal layer \\
\hline $0.13-\mathrm{mm}$ dielectric & L4: Ground plane \\
\hline $0.5-\mathrm{mm}$ dielectric & L5: Signal layer \\
& L6: Connector and surface pads \\
\hline
\end{tabular}

Fig 7. Layer stack up of the test board

The six-layer circuit board is of dimension $7.6 \mathrm{~cm} \times 5 \mathrm{~cm}$. In the test board Power and Ground planes are located on layer 3 and layer 4 with a spacing between them, $0.05 \mathrm{~mm}$. Signals are routed on layer 2 and layer 5. The components consists of one 50-MHz oscillator, one bulk decoupling capacitor, eight octal clock buffers, 28 load capacitors and 32 local decoupling capacitors. A 50-MHz oscillator drives the input pins of one of the octal clock buffers. The other buffers are driven by the first octal clock buffers. A subminiature type A coaxial connector is used to connect to the power supply $3.3-\mathrm{V}$ power to the printed circuit board. The $1-\Omega$ resistors are connected in series with the four pins of one of the buffers, making it possible to measure the power currents to this buffer.

The test hoard is a 6-layer board using CMOS technology. The stack-up of the board is shown in Table I and the layout is shown in Figure 7. The large number of signal nets in this design makes it difficult to visually identify potential EMC problem.

Table - I Details of the stack-up of the Test Board

\begin{tabular}{|l|c|c|c|}
\hline Layer & Material & Thickness & $E_{\mathrm{r}}$ \\
\hline \multirow{2}{*}{ Top } & Metal & $1.2 \mathrm{mils}$ & 1.0 \\
& Dielectric & $8.0 \mathrm{mils}$ & 4.5 \\
\hline \multirow{2}{*}{ GND } & Metal & $1.2 \mathrm{mils}$ & 1.0 \\
& Dielectric & $8.0 \mathrm{mils}$ & 4.5 \\
\hline \multirow{2}{*}{ VDD } & Metal & $1.2 \mathrm{mils}$ & 1.0 \\
& Dielectric & $8.0 \mathrm{mils}$ & 4.5 \\
\hline Bottom & Metal & $1.2 \mathrm{mils}$ & 1.0 \\
\hline
\end{tabular}

\section{BoARD ANALYSIS USING THE DIFFERENTIAL MODE RADIATION ALGORITHM}

The algorithm was first applied to the test board when the buffers were not loaded. The parameters needed to calculate the radiation by the differential mode radiation algorithm and their values are listed in Table II.

Table II .Parameters needed Differential mode algorithm

\begin{tabular}{|c|c|c|}
\hline Parameter & Description & Value \\
\hline$L$ & Boand length & $7.6 \mathrm{~cm}$ \\
\hline$W$ & Beard width & $5.0 \mathrm{~cm}$ \\
\hline$h$ & Space between power planes & $0.05 \mathrm{~mm}$ \\
\hline$c_{t}$ & Dielectric permittivity & 3.88 \\
\hline$R_{c}$ & Average resistance of the components & $0.3 \mathrm{ohms}$ \\
\hline$L_{t}$ & Average inductance of the components & $1.4 \mathrm{nH}$ \\
\hline$h_{t}$ & Rise/fall time of the current signal & $1.5 \mathrm{~ns}$ \\
\hline$t_{2}$ & Rise/fall time of the current signal & $1.5 \mathrm{~ns}$ \\
\hline$T$ & Period of the signal & $20 \mathrm{~ns}$ \\
\hline$C_{P D}$ & Power dissipation capacitance & $20 \mathrm{pF} / \mathrm{gate}$ \\
\hline
\end{tabular}

The first layout is designed without any EMC consideration. Once the component footprints are finalized, the auto-routing algorithm of the PCB layout software carries out routing of the inter-connecting tracks automatically. Auto-routing functions of most PCB layout tools usually take care of the physical aspects such as looking for the most direct and convenient paths amongst the components, but neglect the EMI control aspect. Because of that, the layout tools tend to overlook the return current paths for $+5 \mathrm{~V}$ and signal tracks. One of these typical finished layouts is given in figure 8 . The dark and light lines are interconnecting tracks on upper and lower sides of the PCB, respectively. The $0 \mathrm{~V}$ and $9 \mathrm{~V}$ terminals are for connection to an external $9 \mathrm{~V}$ battery. All the components are placed on the upper side of the PCB and the grey lines are their positions. The components, RG1, U1, U2, and U3 etc., are labeled in accordance with the

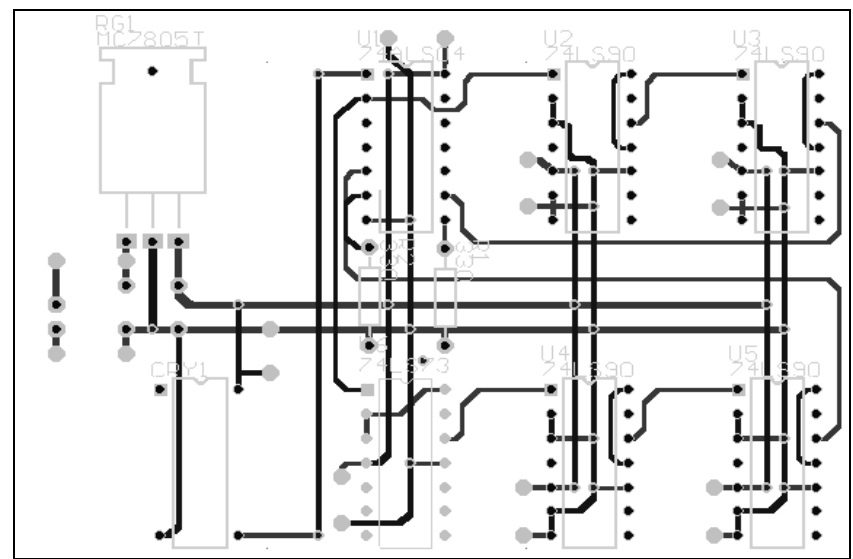

Fig.8 PCB layout for differential mode consideration

standard digital circuit. Since currents flow in closed loops, with no EMC consideration, the layout in figure 8 creates many unexpected large $+5 \mathrm{~V}$-ground and signal-ground loops. Figure 9 shows that radiated emissions of the PCB in frequency range $300-400 \mathrm{MHz}$ have exceeded the CISPR 22 Class B limit marginally. 


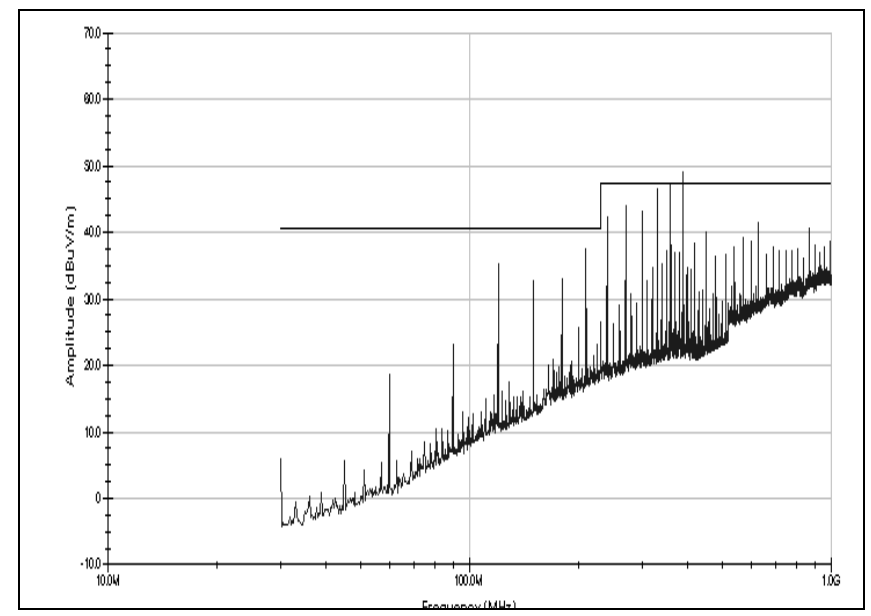

Fig. 9 Radiated emissions for PCB layout

To show the possible impact of $\mathrm{CM}$ radiation of the $\mathrm{PCB}$, a $30 \mathrm{~cm}$ wire is soldered at the far end PCB ground, directly opposite the $9 \mathrm{~V}$ source.

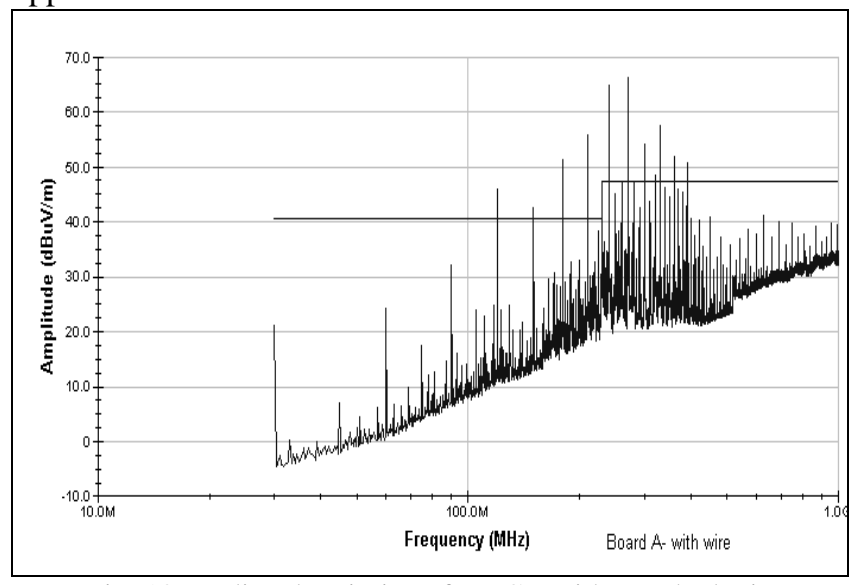

Fig. 10 Radiated emissions for PCB with attached wire

Figure 10 shows the radiated emissions of the PCB with the attached wire. It indicates clearly that the added CM radiation due to the attached wire pushes the emissions up significantly and now the highest emission exceeded the limit by as much as $20 \mathrm{~dB}$. The high $\mathrm{CM}$ radiation is due to the large ground bounce on the PCB ground resulting from poor layout.

\section{CONCLUSION}

Although both of the layout problems identified by the EMC expert software system might have been obvious to an EMC engineer who was familiar with the board and the signals on each of these nets, a lot of effort would have been required to initially locate these problems manually. If changes were made to the layout, this effort would have to be repeated to ensure that no new problems were created. The expert system algorithms are designed to help both experts and non-experts find major potential problems early in the design process without manually examining every net routed on the board.

For more than 40 years, researchers have been pursuing the development of measurement methods, prediction tools, and design techniques for improving the EMC performance of ICs. Looking to the future, there are many difficult challenges ahead, most without clear solutions. A set of issues was highlighted, regarding low-emission design techniques, high-immunity guidelines, extremely high-frequency measurement techniques, as well as efficient methodologies for reliable prediction of EMC performance prior to manufacture. The field of EMC at the component level has grown much beyond the expectations of one or two decades ago. EMC expertise has successfully weathered and, in fact, thrived on the IC technology scale-down. We expect component-level EMC to continue to be an active field of study in the future, as ICs become larger, denser, and operate at higher clock speeds and lower supply voltages. We hope this paper provides readers with a sense of the past and future trends in EM compliance of ICs, following the extraordinary advances of the microelectronics industry.

\section{REFERENCES}

[1] H. Shim, T. Hubing, T. Van Doren, R. DuBroff, J. Drewniak, D. Pommerenke, and R. Kaires, "Expert system algorithms for identifying radiated emission problems in printed circuit boards," in Proc. IEEE Int. Symp. Electromagn. Compat., Santa Clara, CA, vol. 1, Aug. 9-13, 2004, pp. 57-62.

[2] H. Shim and T. Hubing, "Model for estimating radiated emissions from a printed circuit board with attached cables driven by voltage-driven sources," IEEE Trans. Electromagn. Compat., vol. 47, no. 4, pp. 899-907, Nov. 2005.

[3] H. Shim and T. Hubing, "Derivation of a closed-form approximate expression for the self-capacitance of a printed circuit board trace," IEEE Trans. Electromagn. Compat., vol. 47, no. 4, pp. 1004-1008, Nov. 2005.

[4] D. Hockanson, J. Drewniak, T. Hubing, F. Sha, and M. Wilhelm, "Investigation of fundamental EMI source mechanisms driving common-mode radiation from printed circuit boards with attached cables," IEEE Trans. Electromagn. Compat., vol. 38, no. 4, pp. 557-565, Nov. 1996.

[5] H. Shim, Y. Fu, and T. H. Hubing, "Radiated emissions from populated printed circuit boards due to power bus noise," in Proc. IEEE Int. Symp. Electromagn. Compat., Santa Clara, CA, vol. 2,Aug. 9-13, 2004, pp. 396- 400 .

[6] H. Shim and T. Hubing, "A closed-form expression for estimating radiated emissions from the power planes in a populated printed circuit board," IEEE Trans. Electromagn. Compat., vol. 48, no. 1, pp. 74-81, Feb. 2006

[7] F. Schnieder and W. Heinrich, "Model of thin-film microstrip line for circuit design," IEEE Trans.Microw. Theory Tech., vol. 49, no. 1, pp. 104-110, Jan. 2001.

[8] J. Fan, W. Cui, J. L. Drewniak, T. P. Van Doren, and J. L. Knighten, "Estimating the noise mitigation effect of local decoupling in printed circuit boards," IEEE Trans. Adv. Packag., vol. 25, no. 2, pp. 154-165, May 2002.

T.Pearson received B.E. and M.E. degrees in electronics engineering from Bharathiar University, Coimbatore, India. He is currently working towards Ph.D in Electronics and Communication Engineering from Bharath University, Chennai, India. He is currently a teaching faculty in SMK Fomra Institute of Technology. He is life member ISTE. His research interests include the study of electromagnetic interference effects on CMOS digital circuits and digital circuits on VLSI systems particularly device modeling and circuit design.

M. Madheswaran is with Muthyammal Engineering College, Rasipuram, Nammakal District, Tamilnadu, India. He has obtained his Ph.D. degree in Electronics Engineering from Institute of Technology, Banaras Hindu University, Varanasi in 1999. He obtained his M.E. degree in Microwave Engineering from Birla Institute of Technology, Ranchi, Bihar (Now Jarhand). He is a member of IEEE, SPIE, IETE, ISTE, VLSI Society of India and Institution of Engineers(India).He is the Chairman, IEEE India Electron Devices Chapter for the year 2008 \& 2009 and Vice Chairman IEEE India Solid State Circuits Chapter. 